\title{
SOIL MOISTURE RETRIEVAL UNDER VEGETATION: VALIDATION ON TERENO OBSERVATORIES
}

\author{
Thomas Jagdhuber ${ }^{1}$, Miguel Kohling ${ }^{1}$, Irena Hajnsek ${ }^{1,2}$, Konstantinos P. Papathanassiou ${ }^{1}$ \\ ${ }^{1}$ German Aerospace Center, Microwaves and Radar Institute, PO BOX 1116, 82234 Wessling \\ ${ }^{2}$ ETH Zurich, Institute of Environmental Engineering, Schafmattstr. 6, 8093 Zurich \\ Email: thomas.jagdhuber@dlr.de, miguel.kohling@dlr.de, irena.hajnsek@dlr.de, kostas.papathanassiou@dlr.de
}

\begin{abstract}
The extensive field data base of the TERENO project is utilized to validate inversion results for soil moisture under vegetation within two terrestrial observatories (Central German Lowland Observatory and Lower Rhine Valley Observatory). The recently developed hybrid decomposition and inversion approach is applied to L-band fully polarimetric SAR data acquired within the TERENO campaign of 2011 by DLR's novel high resolution F-SAR sensor in order to investigate its potential. The soil moisture results state a spatially continuous inversion under vegetation with a RMSE between 3.2vol.\% and 8.2vol.\% for both observatories. Therefore the results are particularly suited for local analyses on the field level in contrast to soil moisture products with low resolution from passive microwave sensors on the catchment level.
\end{abstract}

Index Terms- TERENO, soil moisture estimation, SAR polarimetry, hybrid decomposition, agriculture

\section{INTRODUCTION}

Global change affects all domains of the environment with complex feedback mechanisms from point to catchment scale. Within the TERENO project these long-term effects on terrestrial ecosystems are studied using a multi-sensor synthesis in four terrestrial observatories, distributed over Germany [1]. In these studies long-term hydrological data, like from soil moisture, are needed to calibrate and validate the environmental models for an improved climate change monitoring.

In order to obtain the necessary spatial coverage, a remotebased sensing technique using polarimetric SAR is exploited. Therefore the penetration of a longer wavelength (L-band) is combined with the observation space of SAR polarimetry to extract soil moisture under vegetation cover.

\section{SOIL MOISTURE RETRIEVAL UNDER VEGETATION COVER}

A polarimetric decomposition represents an innovative unmixing technique to separate different scattering contributions occurring within one resolution cell by means of SAR polarimetry [2]. In natural environments the scattering contributions of the vegetation and the soil need to be decomposed for analysis of the soil moisture conditions.

In [3] a novel hybrid decomposition is presented for a physically-constraint decomposition and inversion of soil moisture under vegetation cover. In this approach the advantages of model-based and eigen-based decompositions are incorporated in one algorithm together with a physically constraint volume component to retrieve the desired soil moisture under vegetation cover.

\section{EXPERIMENTAL DATA}

The developed inversion algorithm was applied on the data set of the TERENO campaign conducted in May/June 2011 in the Central German Lowland (Bode catchment) and the Lower Rhine Valley (Rur catchment) Observatories. Fullypolarimetric SAR data at L-band were acquired in high resolution of $2 \mathrm{~m} \times 0.5 \mathrm{~m}$ (ground range $\mathrm{x}$ azimuth) by DLR's novel F-SAR sensor over both observatories [4].

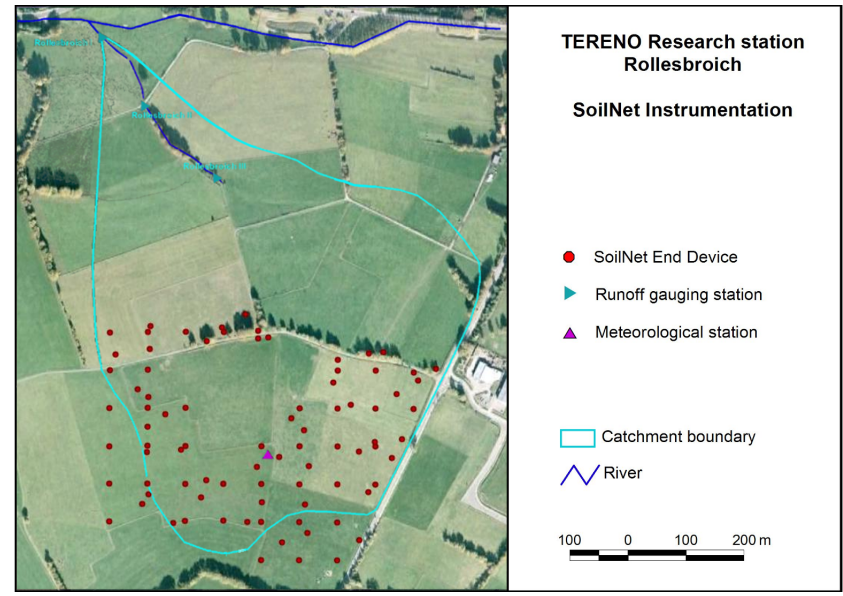

Fig. 1. Wireless soil sensor network (SoilNet) for distributed soil moisture sensing at the Rollesbroich site of the Lower Rhine Valley Observatory, installed and maintained by Forschungszentrum Jülich [5]. Red dots indicate the single probing devices of the SoilNet. 
Simultaneously, soil moisture and vegetation measurements were taken from Forschungszentrum Jülich (FZJ) and Helmholtz Centre for Environmental Research / Water and Earth System Science Competence Center (UFZ/WESS) on selected test fields of both observatories, covering different vegetation and soil types under their respective phenological and hydrological conditions.

A novelty was the incorporation of wireless soil sensor networks (SoilNets) for distributed soil moisture measurements on ground [5]. The SoilNet of Rollesbroich within the Rur catchment is shown in Fig. 1, where the locations of the probing devices, which were available for validation, are marked red in the optical image.

\section{RESULTS FOR SOIL MOISTURE INVERSION WITHIN TERENO OBSERVATORIES}

The results of soil moisture inversion under vegetation cover within the TERENO observatories are shown in Fig. 2 for the Central German Lowland and in Figs. 3-4 for the Rollesbroich and Merzenhausen test site of the Lower Rhine Valley. Forested and urban areas are masked gray considering the invalidity of the polarimetric decomposition and inversion model for these types of scatterers at L-band.
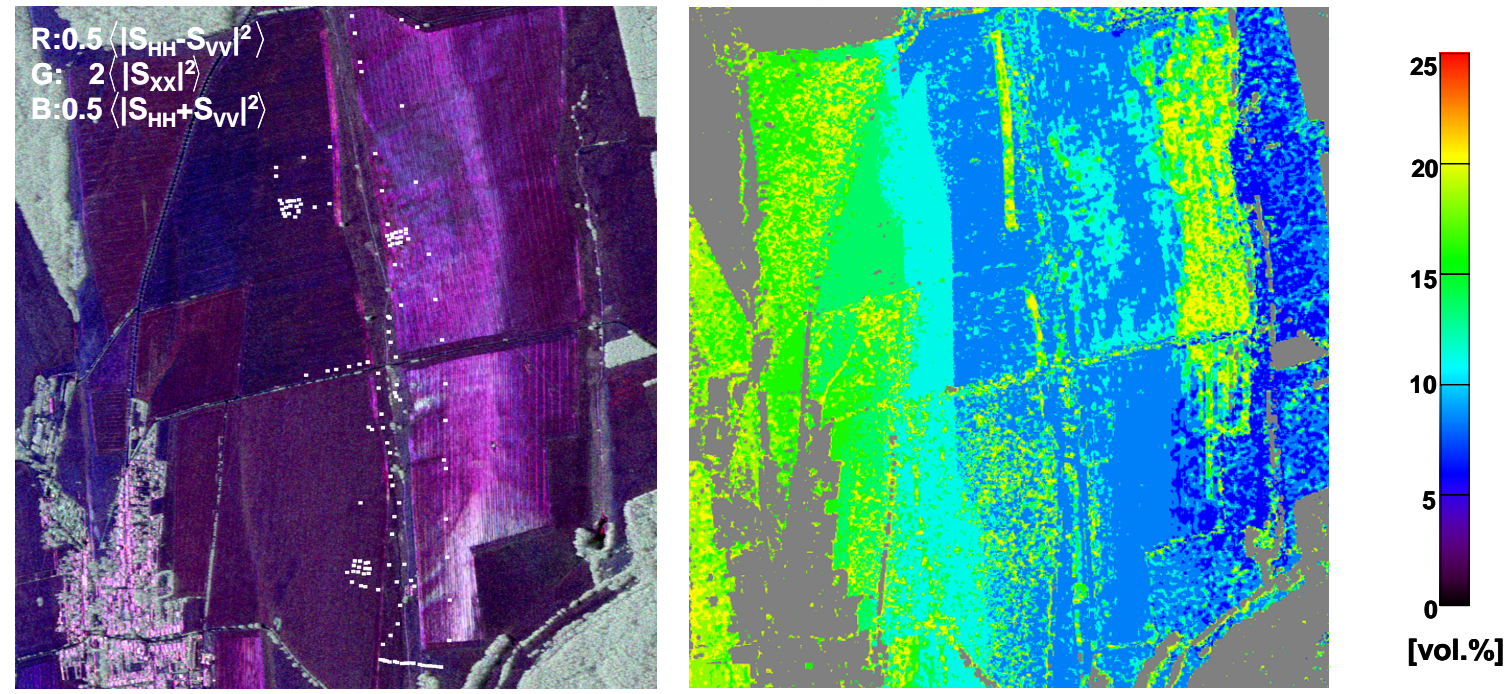

Fig. 2. Pauli-RGB image with measurement points in white [dB] (left) and inverted soil moisture under vegetation cover [vol.\%] (right) for the Bode catchment within the Central German Lowland Observatory; Urban and forested areas are masked gray.
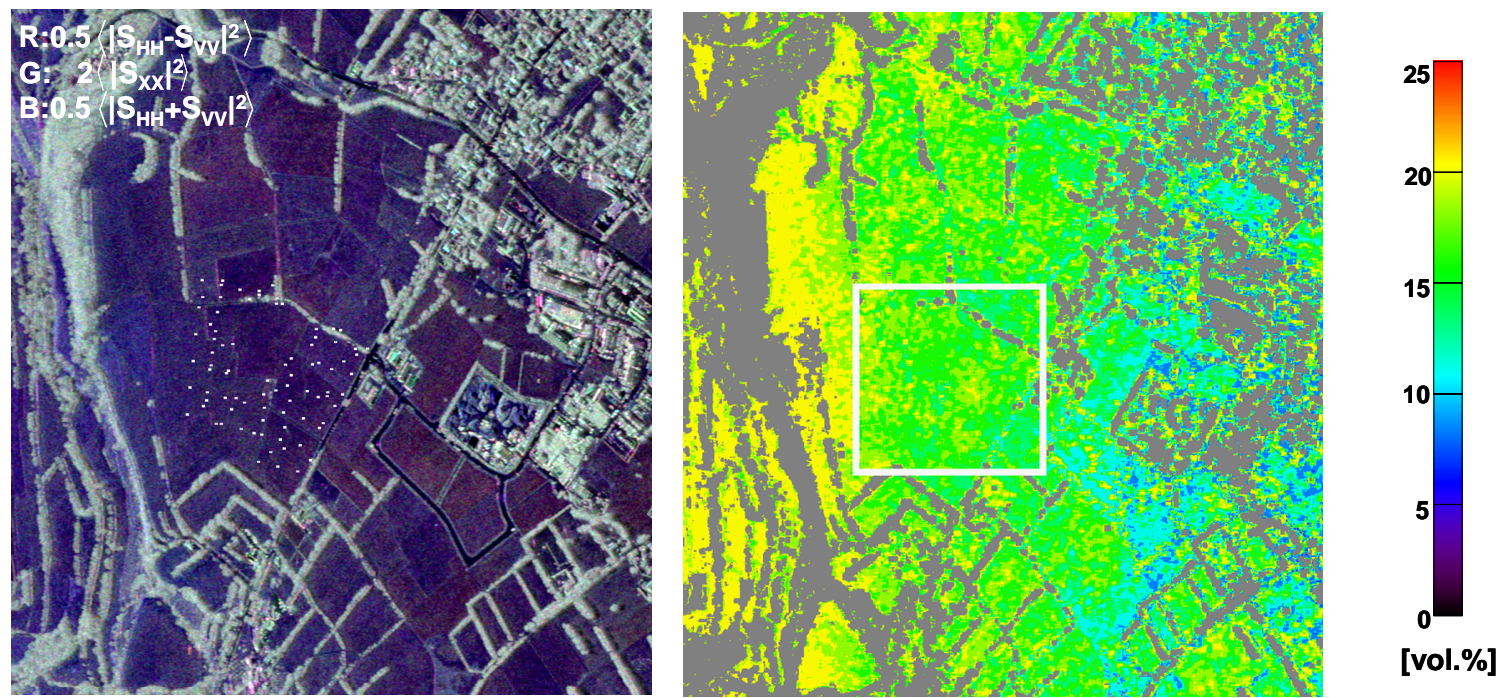

Fig. 3. Pauli-RGB image with measurement points in white [dB] (left) and inverted soil moisture under vegetation cover [vol.\%] (right) for the Rollesbroich test site within the Lower Rhine Valley Observatory; The white frame indicates the maximum extent of the SoilNet (status of spring 2011); Urban and forested areas are masked gray. 

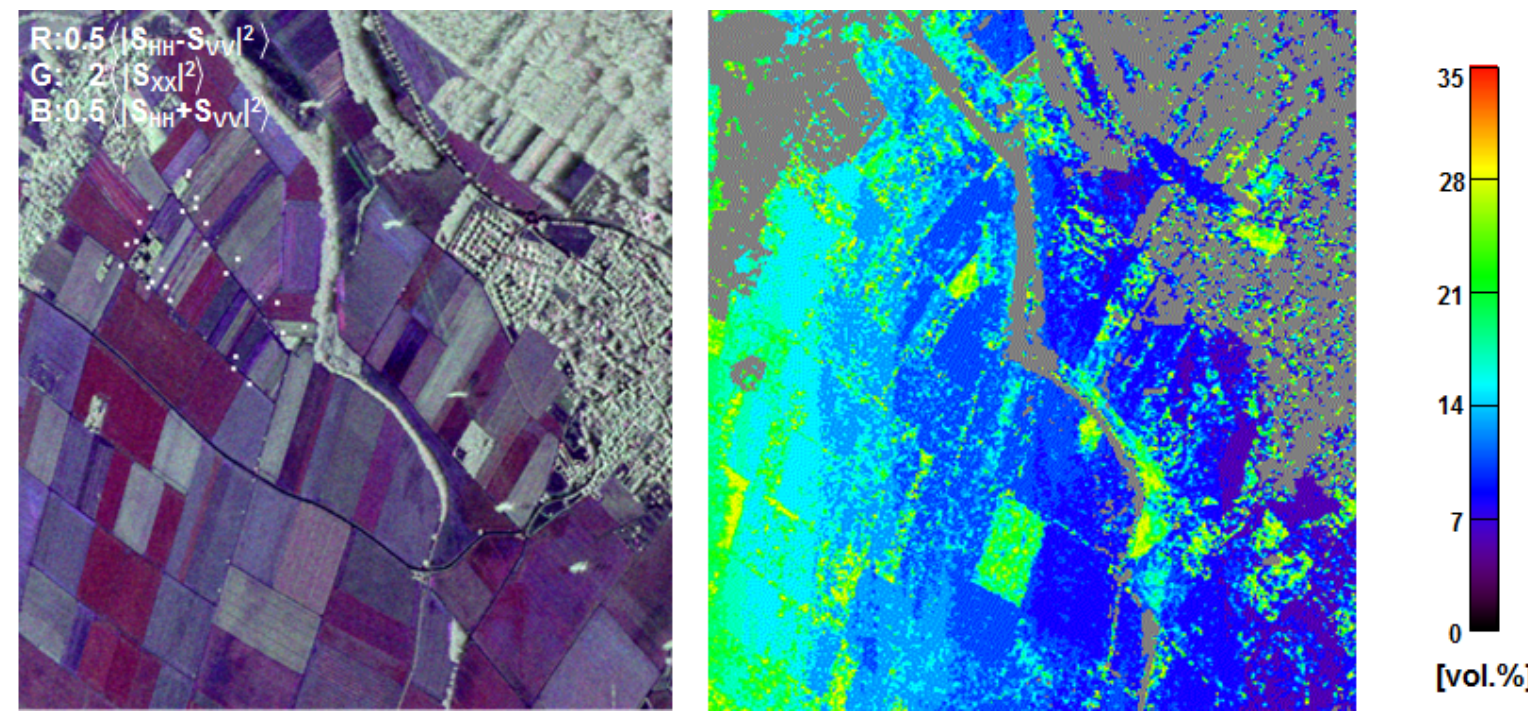

[vol.\%]

Fig. 4. Pauli-RGB image with measurement points in white [dB] (left) and inverted soil moisture under vegetation cover [vol.\%] (right) for the Merzenhausen test site within the Lower Rhine Valley Observatory; Urban and forested areas are masked gray.

For both observatories the soil moisture level ranges predominantly between $5 \mathrm{vol} . \%$ and $15 \mathrm{vol} . \%$, which is verified by the in situ measurements, indicating dry moisture conditions. Due to the very high inversion rate, a spatially continuous image of the soil moisture distribution can be obtained revealing the soil moisture patterns on the test fields. Therefore the decomposition of the soil and the vegetation contributions of the backscattered, polarimetric signature seem to be successful in these cases.

The investigated fields are covered by a variety of crop types (Bode: Winter triticale, winter wheat and summer wheat, grassland; Rollesbroich: Grassland; Merzenhausen: Winter wheat, winter barley, asparagus, sugar beet, potatoes) with vegetation heights from $18-50 \mathrm{~cm}$ (summer wheat, sugar beet, potatoes) to $50-90 \mathrm{~cm}$ (winter wheat, winter barley) [5,6]. Unfortunately, no vegetation measurements are available for the grassland on the campaign dates.

In Fig. 2 the intra-field variability, reflected in the soil moisture patterns, follows mostly the topographical variations on the fields. In order to assess this effects as well as the quality of the soil moisture inversion under vegetation cover, the in situ measurements from the mobile probes (FDR) and from the SoilNet cluster are utilized for comparison with the soil moisture estimates from the decomposed polarimetric SAR data for a validation.

The correlations for the Central Lowland and the Lower Rhine Valley Observatories are shown in Figs. 4-7, respectively. The validation plots state the dry moisture level ranging mainly between 5-15vol.\%, which matches the level of the estimated soil moisture values with an RMSE between 3.2vol.\% and 8.2vol.\%. Outliers of measured data, which deviate more than three standard deviations from the mean value, are canceled for the analyses.
Nonetheless, the dynamics of the estimated soil moisture in Fig. 5 appear rather small for the summer wheat and grassland fields compared with the measured soil moisture values, varying mainly between $5 \mathrm{vol} . \%$ and $15 \mathrm{vol} . \%$. This might be caused by a DEM with a coarse spatial resolution (SRTM90) used for data processing. Consequently, this shortcoming and the low moisture level $(<15 \mathrm{vol}$.\%) requires further analyses, also on other observatories investigated during the TERENO campaign in 2011.

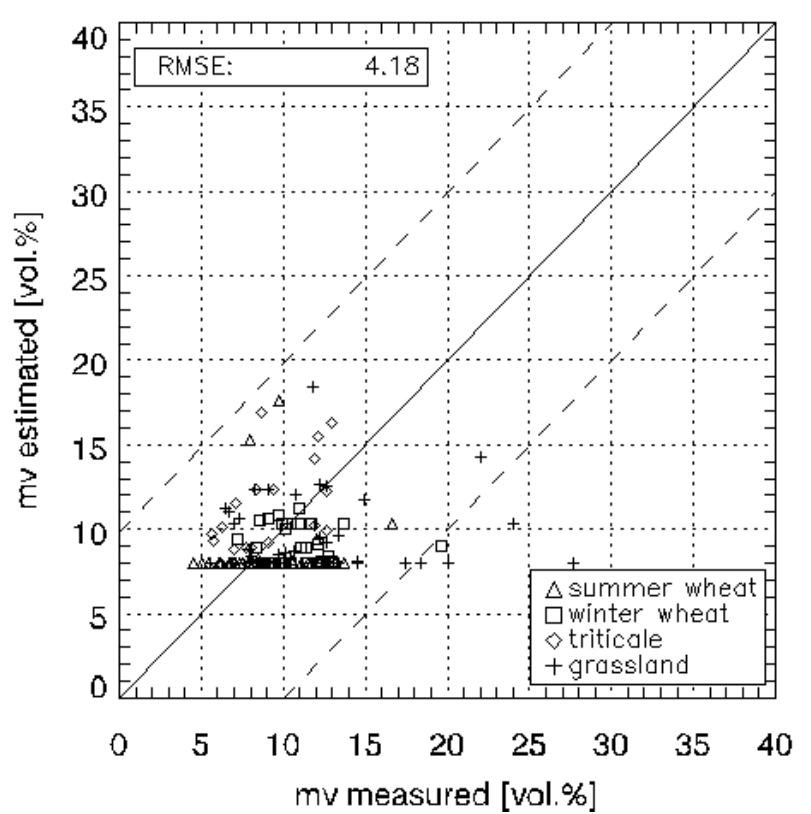

Fig. 5. Correlation of in situ measurements (FDR) with inverted soil moisture values in vol.\% for the Bode catchment within the Central Lowland Observatory; Triangle=summer wheat, square= winter wheat, diamond=winter triticale, plus=grassland. 


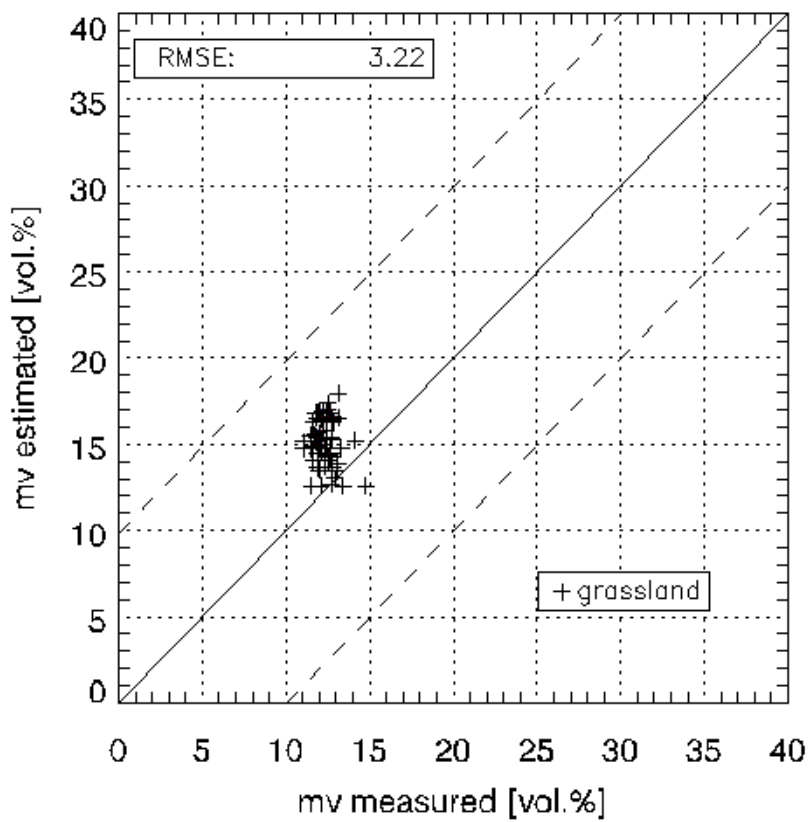

Fig. 6. Correlation of in situ measurements (SoilNet) with inverted soil moisture values in vol.\% for the Rollesbroich test site within the Lower Rhine Valley Observatory; Plus=grassland.

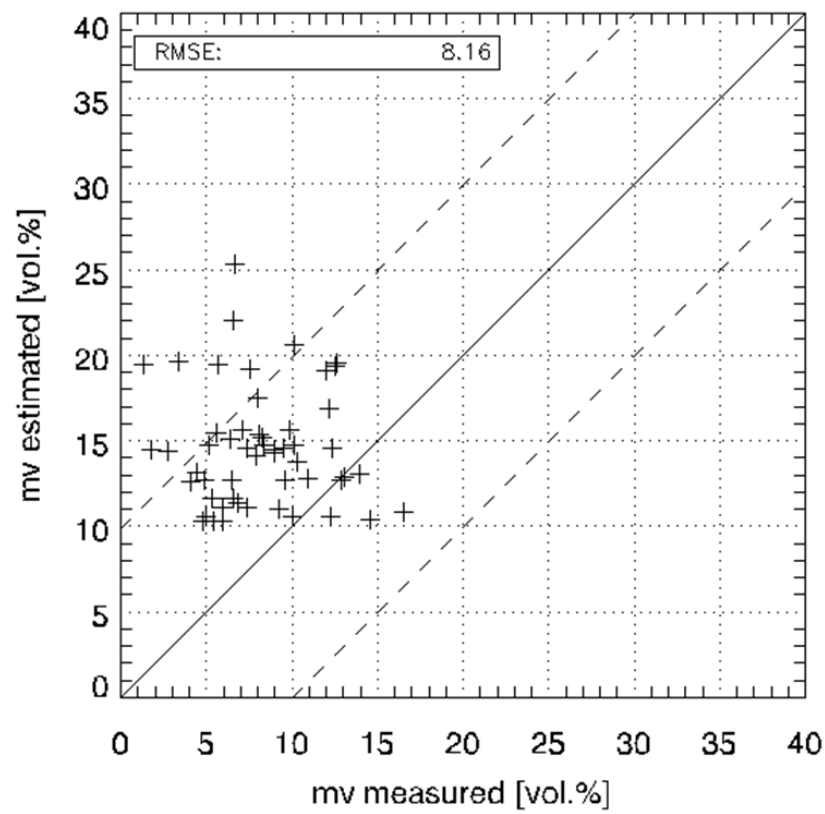

Fig. 7. Correlation of in situ measurements (FDR) with inverted soil moisture values in vol.\% for the Merzenhausen test site within the Lower Rhine Valley Observatory; Plus=randomly sampled measurements for a variety of land use (winter wheat, winter barley, asparagus, sugar beet, potatoes).

\section{SUMMARY AND CONCLUSION}

The hybrid decomposition and inversion algorithm has been applied to the F-SAR fully polarimetric L-band data of the Central Lowland and the Lower Rhine Valley
Observatories. A spatially continuous inversion is obtained with an inversion rate of almost $100 \%$ on the agricultural (non-masked) areas. The intra-field variability of the soil moisture is clearly recognizable due to the high resolution of the SAR data, which are particularly suited for local investigations on the intra-field level compared to data of passive microwave sensors with distinctively lower resolution.

The validations with the mobile FDR probes as well as with the SoilNet cluster result in a RMSE from 3.2vol.\% until 8.2vol.\%, which confirms the potential of the developed soil moisture retrieval algorithm for a dry moisture scenario $(<15 \mathrm{vol} . \%)$. However, the algorithm needs also to be tested for high soil moisture scenarios and different phenological stages in further observatories, including the upcoming TERENO measurement campaign of 2012, which will include all four TERENO observatories.

\section{ACKNOWLEDGEMENTS}

The authors would like to acknowledge the TERENO partners (Forschungszentrum Jülich (FZJ), Helmholtz Centre for Environmental Research/ Water and Earth System Science Competence Center (UFZ/WESS)) for acquisition of the field measurements and the Helmholtz association for supporting the activities.

\section{REFERENCES}

[1] H. Vereecken, “TERENO-Terrestrial Environmental Observatories,” TERENO Kick-off workshop, Berlin, Germany, September 22, 2008.

[2] S.R. Cloude and E. Pottier, "A Review of Target Decomposition Theorems in Radar Polarimetry," IEEE Transactions on Geoscience and Remote Sensing, vol. 34, pp. 498518, 1996.

[3] T. Jagdhuber, I. Hajnsek, K.P. Papathanassiou and A. Bronstert, "A Hybrid Decomposition for Soil Moisture Estimation under Vegetation Cover Using Polarimetric SAR," Proc. of the $5^{\text {th }}$ International Workshop on Science and Applications of SAR Polarimetry and Polarimetric Interferometry, ESA-ESRIN, Frascati, Italy, January 24-28, p.1-6, 2011.

[4] T. Jagdhuber, M. Kohling, I. Hajnsek, "TERENO F-SAR Airborne Campaign 2011 @ Rur, Bode and Ammer catchments," CT Environmental Sensing Meeting, DLR Oberpfaffenhofen, Germany, November 29, 2011.

[5] C. Montzka, H. Bogena, S. Hasan, W. Qu, R. Baatz, "Measurements in the Rur catchment 2011," CT Environmental Sensing Meeting, DLR Oberpfaffenhofen, Germany, November 29, 2011.

[6] M. Pause, "Ground-based activities during airborne EnvSens campaigns 2011 - TERENO Central German Lowland Observatory," CT Environmental Sensing Meeting, DLR Oberpfaffenhofen, Germany, November 29, 2011. 